\section{Health Consequences of Aircraft Noise}

by Prof. Dr. med. Martin Kaltenbach et al. in volume 31-32/2008

\section{Noise Research is Hampered}

Kaltenbach et al are of the opinion that relations between external influences and negative effects—such as illness, sleeping disorders, and learning disorders - can be shown only by means of epidemiological studies. This statement is not true as it is exclusive and hampers research into the physiological effects of noise. Kaltenbach et al generalize; although the authors of all cited studies are cautious in interpreting their results, which rarely reach significance and are sometimes inconsistent. No critical discussion and evaluation of the cited studies has taken place.

The discussion on the effects of nocturnal noise does not reflect the current state of knowledge. The average noise levels that almost all epidemiological studies are based on are not equivalent in terms of effect. For a long time now, the most important organizations that are active in noise protection (BUND, the federal association against railway noise, the German National Organization Against Aircraft Noise, the German Working Group for Noise Abatement, and Verkehrsclub Deutschland (VCD, an association for sustainable mobility and a major German transport and environmental organisation) have been demanding that attention be paid to maximum noise levels and to how often these are reached. It is not true that the criticized synopsis uses awakening reactions - which Kaltenbach et al regard as of little relevance — as the basis for its evaluation; it uses the derived cortisol concentrations as a possible link to long term health impairments. The noise limits that the authors present in the subjunctive are not based on scientific criteria as per the literature. Noise can without question result in health impairments; as a possible ubiquitous stressor it should therefore be reduced. The intention of the synopsis is to make a contribution to this-based on causal connections that are justified by effects-but so does the aircraft noise law. DOl: 10.3238/arztebl.2009.0071a

\section{REFERENCES \\ 1. Kaltenbach M, Maschke C, Klinke R: Health Consequences of Aircraft Noise. Dtsch Arztebl Int 2008; 105(31-32): 548-56.}

Prof. Dr. Manfred Spreng

Lange Zeile 121, 91054 Erlangen, Germany

\section{Conflict of interest statement}

The author acts as an expert and gives presentations for airport expansions, for those affected by railway noise, and for state measures.

\section{In Reply:}

Our article says that "Laboratory tests are of only limited value." This is not a statement of exclusion. With regard to the development of hypertension, studies in sleep laboratories do not permit reliable conclusions about chronic diseases.

The meaningfulness of any study has its limits. To deduce guide values, the results of all meaningful studies have to be evaluated. That is exactly what we did.

All studies of hypertension have shown significant results, as shown in table 1 . The odds ratios reported there reach significance if the accompanying confidence interval does not include 1 . This is the case in nine studies. The results of the Aydin study also reach significance.

The 2001 "synopsis," of which Spreng was a co-author and which was commissioned by Fraport, says that "the basis for the assessment (of the night) is the avoidance of additional noise related awakening." In their 2007 article, the authors of the "synopsis" do not "see any reason to deviate from earlier findings." Only in the context of the expansion of Frankfurt airport, a cortisol model by Spreng was cited, to provide a reason for the preventive guide value. To date, the validity of this models has not been verified.

Acute sleep disruption (awakening) due to brief noise events ( $<60$ seconds) can be predicted more precisely on the basis of the maximum noise level than the constant volume. However, awakening reactions are not appropriate for a health assessment that includes chronic diseases. Avoiding awakening reactions is not sufficient to prevent chronic health impairments. One of the article's main intentions is to raise attention for the fact that these recent research results have to be borne in mind.

The guide values in table 3 were deduced by using toxicology results. The lowest level of the first significant noise level class was interpreted as "no observed adverse effect level" and assigned to the preventive guide value. The top level of the first significant noise level class was interpreted as "lowest observed adverse effect level" and assigned to the critical tolerance value.

The "synopsis" does not show which method the authors used to gain their guide values. Further, it is not clear whether the "synopsis" underwent a similar peer review procedure as the article published in the Ärzteblatt. It is not enough to rely on earlier research resultsthe results of more recent studies will always have to be taken into account. This is the case especially for chronic health impairments that cannot be reliably identified by using laboratory results only. D0l: 10.3238/arztebl.2009.0071b

\section{REFERENCES \\ 1. Kaltenbach M, Maschke C, Klinke R: Health Consequences of Aircraft Noise. Dtsch Arztebl Int 2008; 105(31-32): 548-56.}

Unfortunately, our co-author Rainer Klinke has passed away in the meantime.

Prof. Dr. med. Martin Kaltenbach; PD Dr. Ing. Christian Maschke Address for correspondence: Falltorweg 8, 63303 Frankfurt, Germany martinkaltenbach@arcor.de

Conflict of interest statement

The authors of both the letter and the reply declare that no conflict of interest exists according to the guidelines of the International Committee of Medical Journal Editors. 